\title{
Slow-roll versus stochastic slow-roll inflation
}

\author{
Z. Haba ${ }^{\mathrm{a}}$ (1) \\ Institute of Theoretical Physics, University of Wroclaw, 50-204 Wroclaw, Poland
}

Received: 5 June 2019 / Accepted: 18 October 2019 / Published online: 10 November 2019

(c) The Author(s) 2019

\begin{abstract}
We consider the classical wave equation with a thermal and Starobinsky-Vilenkin noise which in the slowroll and long wave approximation describes the quantum fluctuations of the gravity-inflaton system in an expanding metric. We investigate the resulting consistent stochastic Einstein-Klein-Gordon system in the slow-roll regime. We show in some models that the slow-roll requirements (of the negligence of $\partial_{t}^{2} \phi$ ) can be satisfied in the probabilistic sense for the stochastic system with quantum and thermal noise for arbitrarily large time and an infinite range of fields. We calculate expectation values of some inflationary variables taking into account quantum and thermal noise. We show that the mean acceleration $\left\langle\partial_{t}^{2} a\right\rangle$ can be negative or positive (depending on the model) when the random fields take values beyond the classical range of inflation.
\end{abstract}

\section{Introduction}

In the standard classical approach to inflation the slow-roll approximation is very useful. Fortunately, it applies just in the range of field configuration where it is needed,i.e., during the time of the accelerated expansion. However, in the description of the early stages of the universe evolution quantum and thermal fluctuations are unavoidable. With the fluctuations the usual criteria of the slow-roll strictly speaking do not apply. In fact such fluctuations can lead to an eternal inflation, i.e. the inflation never ends as suggested first by Linde [1]. We are going to investigate the quantum and thermal fluctuations quantitatively on the basis of differential equations which are supposed to describe the exact time evolution at least at the early stages. Our approach is based on the Starobinsky approach [2] to quantum fluctuations and the description of thermal fluctuations known from the theory of Brownian motion [3] and applied to inflation in [4-8]. Starobinsky [2] suggested an approach which treats quantum scalar field

\footnotetext{
a e-mail: zbigniew.haba@uwr.edu.pl
}

non-perturbatively in classical Einstein equations. The idea is based on an earlier observation [9-12] that the quantum scalar field in an expanding universe behaves as a classical diffusion process. In such a case we obtain a stochastic Einstein-Klein-Gordon (EKG)system. In the slow-roll limit the stochastic EKG system is approximated by a first order differential equation of a diffusion process. The approximation applies well for deterministic systems in the range of slow-roll. If we restrict the range of evolution of $\phi$ in the stochastic equation in order to cut it to the classical range of inflation then we must introduce boundary conditions as is done in $[13,14]$. However, the stochastic equation makes sense in an unbounded field configuration space till a random explosion time. If we calculate this conditional probability distribution then it satisfies the usual Fokker-Planck equation. We prefer the approach based on an unbounded field evolution. On the basis of the Fokker-Planck equation we study the problem of probabilistic estimates of the error of the slow-roll approximation. We hope that as in the theory of the Brownian motion of a particle in the medium with a friction the first order equation well approximates the second order stochastic non-linear oscillator equation [3].

The plan of the paper is the following. In Sect. 2 we recall the basic equations of the inflaton model. We describe the standard slow-roll approximation in Sect. 3. The diffusion process corresponding to slow-roll approximation is discussed in Sect. 4. In Sect. 5 we describe some models of the inflaton potential and calculate the scale factor as a function of the field. In Sect. 6 the slow-roll approximation is discussed on the basis of the probability distribution of the inflaton and its time derivatives. The aim is to set a framework which allows to estimate corrections to the slow-roll. In Sect. 7 we review standard results of the theory of diffusion processes in order to explain what happens with solutions of the inflaton models. In Sect. 8 we calculate the stationary probability distribution of the inflaton diffusion process. In the main Sect. 9 of this paper we calculate some large time expectation values using the stationary probability distribu- 
tion (this section can be considered as an application of the methods and results of [15]). In the Appendix we explain the minor changes which come out from a change of time (from cosmic time to the e-fold time).

\section{Inflaton wave equation}

In the standard approach to inflation [16] we consider a flat expanding background metric

$d s^{2}=g_{\mu \nu} d x^{\mu} d x^{\nu}=d t^{2}-a^{2} d \mathbf{x}^{2}$

Accelerated expansion is generated by the scalar field satisfying a non-linear wave equation (where $H=a^{-1} \partial_{t} a$ )

$\partial_{t}^{2} \phi-a^{-2} \triangle \phi+3 H \partial_{t} \phi+V^{\prime}(\phi)=0$

The expansion scale $a$ is determined by the Friedman equation

$H^{2}=\frac{8 \pi G}{3}\left(V+\frac{1}{2}\left(\partial_{t} \phi\right)^{2}\right)$

We are going to neglect $\partial_{t}^{2} \phi$ in Eq. (1). Hence, we require $\left|\partial_{t}^{2} \phi\right|<<\left|3 H \partial_{t} \phi\right|$. This condition is satisfied if

$\tilde{\epsilon}=\frac{1}{16 \pi G}\left(V^{\prime}\right)^{2} V^{-2}$

and

$\tilde{\eta}=\frac{1}{8 \pi G} V^{\prime \prime} V^{-1}$

are small. We calculate

$\partial_{t}^{2} a=\frac{a^{2} H^{3}}{\partial_{t} a}(1-\tilde{\epsilon})=a H^{2}(1-\tilde{\epsilon})$

(differentiating Eq. (2) with the neglect of $\partial_{t}^{2} \phi$ ). We have an acceleration if $\tilde{\epsilon}<1$.

\section{Stochastic Einstein-Klein-Gordon system}

We consider a modified version of Eq. (1)

$$
\begin{aligned}
\partial_{t}^{2} \phi-a^{-2} \triangle \phi & +\left(3 H+\beta \gamma^{2}\right) \partial_{t} \phi+V^{\prime}(\phi) \\
& +\frac{3}{2} \beta \gamma^{2} H \phi=\eta .
\end{aligned}
$$

Equation (6) is to describe the inflaton in the long wave limit and includes a noise $\eta$ resulting from quantum fluctuations of Refs. [2,9-12] as well as from the random interaction with the environment at the temperature $\beta^{-1}$ introduced in $[17,18]$ ( $\beta$ has been omitted in [18]). $\beta \gamma^{2}$ is the friction coming from the environment (in [17] $\beta \gamma^{2}$ is denoted as $\gamma^{2}$ ). In [17] Eq. (6) is derived under different assumptions (especially in Appendix $B$ of Ref. [17]) than in [18]. With our assumptions concerning the masses and couplings of the environmental fields [18] we obtain Eq. (6) in the long wave limit (the short wave limit of the environmental noise in an expanding universe will be discussed in [19]). With both random fluctuations the noise $\eta$ on the rhs of Eq. (6) takes the form

$\eta \equiv \partial_{t} \xi=\gamma a^{-\frac{3}{2}} \partial_{t} B+\frac{3}{2 \pi} H^{\frac{5}{2}} \partial_{t} W$

where the first term is the thermal noise. The second term describes the diffusive behaviour of the high frequency part of the inflaton in an expanding universe. The factor $a^{-\frac{3}{2}}$ in the thermal noise comes from the metric weight det $\left|g_{\mu \nu}\right|^{\frac{1}{4}}$. The factor $\frac{3}{2 \pi} H^{\frac{5}{2}}$ is chosen in order to reproduce the correlation functions of the quantum scalar field in an expanding universe [20]. We express the thermal noise by the Brownian motion $B$. This is a continuous Gaussian process whose derivative has the covariance

$\left\langle\partial_{t} B \partial_{s} B\right\rangle=\delta(t-s)$

$\partial_{t} W$ (in Eq. (7)) is an independent Gaussian stochastic process with the same covariance (8).

With the thermal noise we would violate the conservation law for the scalar energy-momentum $T_{\mu \nu}(\phi)$. If $\left(T^{0 v}(\phi)\right)_{; v}=Q$ then we introduce a compensating dark energy density $\rho_{d}$ such that $\partial_{t} \rho_{d}+3 H(w+1) \rho_{d}=-Q$ with $w=-1$ (then $\rho_{d}=-\int d t Q$ see [15] for more details). Then, Eq. (2) still remains true in a differential form. In such a case we obtain a closed random dynamical system (in the first order form) with the Starobinsky-Vilenkin noise $W$ and the environmental noise $B$ [15]

$$
\begin{aligned}
d \phi= & \Pi d t, \\
d \Pi= & -\left(3 H+\beta \gamma^{2}\right) \Pi d t-V^{\prime} d t-\frac{3}{2} \beta \gamma^{2} H \phi d t \\
& +\gamma a^{-\frac{3}{2}} \circ d B+\frac{3}{2 \pi} H^{\frac{5}{2}} \circ d W, \\
d H= & -4 \pi G \Pi^{2} d t, \\
d a= & H a d t .
\end{aligned}
$$

with Eq. (11) replacing Eq. (2). We interprete the stochastic equations in the Stratonovitch sense and use the o-notation for Stratonovitch differentials following Ref. [21]. From Eq. (12)

$$
\ln \left(\frac{a}{a_{0}}\right)=\int_{0}^{t} H d s
$$

\section{Diffusion equation for the slow-roll inflation}

The program outlined at the end of Sect. 3 to solve the stochastic Einstein-Klein-Gordon (EKG) equations for the $(a, H, \phi, \Pi)$ variables and calculate the probability distribution is difficult to perform in a non-perturbative way. We shall rely on approximations. On the preliminary stage we 
neglect the spatial derivatives in Eq. (1). We omit the noise in Eq. (10) and the $\beta \gamma^{2}$ terms. Then, it follows from Eqs. (9)-(12) that

$H=\sqrt{\frac{8 \pi G}{3}\left(V+\frac{1}{2} \Pi^{2}\right)}$.

and

$a=a_{0} \exp \left(-8 \pi G \int d \phi V\left(V^{\prime}\right)^{-1}\right)$

Using, Eqs. (9)-(12) we can obtain the Fokker-Planck equation for the probability distribution of $(a, \phi, \Pi)$. On a formal level we make the next simplifying assumption $d \Pi \simeq 0$ and $\gamma \simeq 0$ in Eqs. (9)-(12). We justify this approximation (if $H$ is large) in Sect. 5 on the level of probability distributions . Then, the system of Eqs. (9)-(12) is reduced to

$d \phi=-\frac{V^{\prime}}{3 H} d t+\frac{\gamma}{3 H} a^{-\frac{3}{2}} \circ d B+\frac{1}{2 \pi} H^{\frac{3}{2}} \circ d W$,

with explicit functions $H(\phi)$ and $a(\phi)$. The approximation of the stochastic non-linear wave equation (6) by a diffusion equation has been extensively discussed in the theory of Brownian motion [3]. The main arguments for this approximation will be discussed in Sect. 5 .

Equation (16) in the Stratonovich interpretation of the stochastic equations [21] leads to the equation (retrospective Kolmogorov equation) for the transition function $P\left(t, \phi ; s, \phi^{\prime}\right)[22]$

$$
\begin{aligned}
\partial_{t} P= & \frac{\gamma^{2}}{18} \frac{1}{H a^{\frac{3}{2}}} \partial_{\phi} \frac{1}{H a^{\frac{3}{2}}} \partial_{\phi} P+\frac{1}{8 \pi^{2}} H^{\frac{3}{2}} \partial_{\phi} H^{\frac{3}{2}} \partial_{\phi} P \\
& -(3 H)^{-1} V^{\prime} \partial_{\phi} P .
\end{aligned}
$$

and to the adjoint (prospective Kolmogorov or FokkerPlanck) equation for the probability distribution $P\left(s, \phi^{\prime} ; t, \phi\right)$

$$
\begin{aligned}
\partial_{t} P= & \frac{\gamma^{2}}{18} \partial_{\phi} \frac{1}{H a^{\frac{3}{2}}} \partial_{\phi} \frac{1}{H a^{\frac{3}{2}}} P+\frac{1}{8 \pi^{2}} \partial_{\phi} H^{\frac{3}{2}} \partial_{\phi} H^{\frac{3}{2}} P \\
& +\partial_{\phi}(3 H)^{-1} V^{\prime} P .
\end{aligned}
$$

\section{The evolution of the scale factor $a(\phi)$}

The dependence of $a$ on $\phi$ is determined in the slow-roll approximation by Eq. (15). Let us consider some examples of potentials appearing in inflation models [23-26]. For a chaotic inflation [1] $V=g \phi^{2 n}(n \geq 1)$

$a=a_{0} \exp \left(-2 \pi G n^{-1} \phi^{2}\right)$.

If $V=g \exp (\lambda \phi)$ then

$a=a_{0} \exp \left(-\frac{8 \pi G}{\lambda} \phi\right)$.
If $\phi \rightarrow+\infty$ then $a \rightarrow 0$, if $\phi \rightarrow-\infty$ then $a \rightarrow \infty$. For the Starobinsky potential [25]

$V=g(\exp (\lambda \phi)-1)^{2}$

we have

$a=a_{0} \exp \left(-\frac{4 \pi G}{\lambda} \phi-\frac{4 \pi G}{\lambda^{2}} \exp (-\lambda \phi)\right)$

$a(\phi)$ is bounded and $a \rightarrow 0$ if $|\phi| \rightarrow \infty$.

The potential for the "natural inflation" [26] describing the axion inflation can be defined as

$V=g(1+\cos (\lambda \phi))$

From Eq. (15) we obtain

$a=a_{0}\left|\sin \left(\frac{\lambda \phi}{2}\right)\right|^{\frac{16 \pi G}{\lambda^{2}}}$

Here, $-\pi \leq \lambda \phi \leq \pi$.

For the double-well potential

$V(\phi)=\frac{g}{4}\left(\phi^{2}-\frac{\mu^{2}}{g}\right)^{2}$.

$a=a_{0}|\phi|^{\frac{2 \pi G \mu^{2}}{g}} \exp \left(-\pi G \phi^{2}\right)$.

When $|\phi| \rightarrow \infty$ then $a \rightarrow 0$.

In subsequent sections we also consider the double-well in the form

$V=\frac{g_{n}}{4}\left(\phi^{2}-\frac{\mu^{2}}{g}\right)^{n}$

(where $g_{n}$ is a dimensional coupling constant) then

$a=a_{0}|\phi|^{\frac{4 \pi G \mu^{2}}{n g}} \exp \left(-\frac{2 \pi G}{n} \phi^{2}\right)$.

\section{Slow-roll approximation for the probability distribution}

The approximation of Eq. (6) by Eq. (16) can be expressed as an omission of $\partial_{t}^{2} \phi$. For a deterministic system this approximation can be controlled by a solution of the slow-roll equation. In a stochastic system solely estimates of probabilities make sense. We must estimate the probability that $\partial_{t}^{2} \phi$ is small.In the system of differential equations (9)-(12) $a$ is a random variable. In principle, we could write down a partial differential equation for the probability distribution $P$ of $(a, \phi, \Pi)$ and subsequently integrating it over $a$ and $\Pi$ we could reduce it to the probability distribution of $\phi$ (this is the probability of finding $\phi$ no matter what are the values of $a$ and $\Pi$ ). This is however technically difficult to achieve. We follow the approximations of Sects. 4, 5. We assume that $H$ (14) and $a$ (15) are explicit functions of $\phi$ and $\Pi$. The transition function $P_{t}^{r e t}\left(\phi, \Pi ; \phi^{\prime}, \Pi^{\prime}\right)$ of the process (9), (10) 
(neglecting the $\beta \gamma^{2}$ terms, i.e., assuming $\beta \gamma^{2}<<3 H$ ) satisfies the equation (retrospective Kolmogorov equation)

$$
\begin{aligned}
\partial_{t} P^{r e t}= & \frac{\gamma^{2}}{2} a^{-\frac{3}{2}} \partial_{\Pi} a^{-\frac{3}{2}} \partial_{\Pi} P^{r e t} \\
& +\frac{9}{8 \pi^{2}} H^{\frac{5}{2}} \partial_{\Pi} H^{\frac{5}{2}} \partial_{\Pi} P^{r e t}-\left(3 H \Pi+V^{\prime}\right) \partial_{\Pi} P^{r e t} \\
& +\Pi \partial_{\phi} P^{r e t} .
\end{aligned}
$$

The adjoint (Fokker-Planck) equation for $P_{t}\left(\phi^{\prime}, \Pi^{\prime} ; \phi, \Pi\right)$ is

$$
\begin{aligned}
\partial_{t} P= & \frac{\gamma^{2}}{2} \partial_{\Pi} a^{-\frac{3}{2}} \partial_{\Pi} a^{-\frac{3}{2}} P \\
& +\frac{9}{8 \pi^{2}} \partial_{\Pi} H^{\frac{5}{2}} \partial_{\Pi} H^{\frac{5}{2}} P+\partial_{\Pi}\left(3 H \Pi+V^{\prime}\right) P-\partial_{\phi} \Pi P .
\end{aligned}
$$

In Eq. (28)we make a change of variables $(\phi, \Pi) \rightarrow(X, Y)$ where

$$
\begin{aligned}
& X=\phi+\frac{1}{3 H} \Pi \\
& Y=\Pi
\end{aligned}
$$

In new coordinates the transition function (28) reads

$$
\begin{aligned}
\partial_{t} P^{r e t}= & \frac{\gamma^{2}}{2} a^{-\frac{3}{2}}\left(\partial_{Y}+\frac{1}{3 H} \partial_{X}\right) a^{-\frac{3}{2}}\left(\partial_{Y}+\frac{1}{3 H} \partial_{X}\right) P^{r e t} \\
& +\frac{9}{8 \pi^{2}} H^{\frac{5}{2}}\left(\partial_{Y}+\frac{1}{3 H} \partial_{X}\right) H^{\frac{5}{2}}\left(\partial_{Y}+\frac{1}{3 H} \partial_{X}\right) P^{r e t} \\
& -\left(3 H Y+V^{\prime}\right)\left(\partial_{Y}+\frac{1}{3 H} \partial_{X}\right) P^{r e t} \\
& +Y\left(1+Y \partial_{\phi}\left(\frac{1}{3 H}\right)\right) \partial_{X} P^{r e t} .
\end{aligned}
$$

The change of coordinates in the Fokker-Planck equation (29) is

$$
\begin{aligned}
\partial_{t} P= & \frac{\gamma^{2}}{2}\left(\partial_{Y}+\frac{1}{3 H} \partial_{X}\right) a^{-\frac{3}{2}}\left(\partial_{Y}+\frac{1}{3 H} \partial_{X}\right) a^{-\frac{3}{2}} P \\
& +\frac{9}{8 \pi^{2}}\left(\partial_{Y}+\frac{1}{3 H} \partial_{X}\right) H^{\frac{5}{2}}\left(\partial_{Y}+\frac{1}{3 H} \partial_{X}\right) H^{\frac{5}{2}} P \\
& +\left(\partial_{Y}+\frac{1}{3 H} \partial_{X}\right)\left(3 H Y+V^{\prime}\right) P \\
& -\partial_{X}\left(Y\left(1+Y \partial_{\phi}\left(\frac{1}{3 H}\right)\right) P\right) .
\end{aligned}
$$

We wish to get rid off the dependence of $P$ on $Y$ altogether. We impose the slow-roll requirement on the probability distribution $P$ assuming that the dependence (14) of $H$ on $\Pi$ is negligible and we neglect $\partial_{\phi} \frac{1}{H}$ in Eqs. (32), (33) (the assumption that $H$ is slowly varying). In Eqs. (28), (29) the order of derivatives over $\Pi$ and multiplicative factors is not relevant when the dependence of $H$ and $a$ on $\Pi$ is neglected. However, after a change of variables the multiplicative factors become dependent on $X$ and $Y$. When we subsequently perform a limit of large $H$ (slow-roll) then the order of factors becomes significant. So, the approximation has to be performed in a consistent way. In these equations we approximate $V^{\prime}(\phi)=$ $V^{\prime}\left(X-\frac{1}{3 H} Y\right) \simeq V^{\prime}(X), H(\phi)=H\left(X-\frac{1}{3 H} Y\right) \simeq H(X)$ and neglect $\partial_{\phi} \frac{1}{3 H}$. We integrate the transition function over $Y$,i.e., $\int d Y P^{r e t}\left(s, X, Y ; t, X^{\prime}, Y^{\prime}\right) \equiv \hat{P}^{r e t}\left(s, X ; t, X^{\prime}, Y^{\prime}\right)$. Then, the limit of slowly varying $H$ and small $\frac{Y}{H}$ for the integrated (over $Y$ ) transition function is

$$
\begin{aligned}
\partial_{t} \hat{P}^{r e t}= & \frac{\gamma^{2}}{2} a^{-\frac{3}{2}} \frac{1}{3 H} \partial_{X} a^{-\frac{3}{2}} \frac{1}{3 H} \partial_{X} \hat{P}^{r e t} \\
& +\frac{9}{8 \pi^{2}} H^{\frac{5}{2}} \frac{1}{3 H} \partial_{X} H^{\frac{5}{2}} \frac{1}{3 H} \partial_{X} \hat{P}^{r e t} \\
& -V^{\prime} \frac{1}{3 H} \partial_{X} \hat{P}^{r e t}-3 H \hat{P}^{r e t} .
\end{aligned}
$$

The probability distribution of $X$ is $\hat{P}_{t}=\int d Y P\left(s, X^{\prime}\right.$, $\left.Y^{\prime} ; t, X, Y\right)$. It follows that the integral over $Y$ of the FokkerPlanck equation (33) gives

$$
\begin{aligned}
\partial_{t} \hat{P}_{t}= & \frac{\gamma^{2}}{2} \frac{1}{3 H} \partial_{X} a^{-\frac{3}{2}} \frac{1}{3 H} \partial_{X} a^{-\frac{3}{2}} \hat{P}_{t} \\
& +\frac{9}{8 \pi^{2}} \frac{1}{3 H} \partial_{X} H^{\frac{5}{2}} \frac{1}{3 H} \partial_{X} H^{\frac{5}{2}} \hat{P}_{t}+\frac{1}{3 H} \partial_{X}\left(V^{\prime} \hat{P}_{t}\right) .
\end{aligned}
$$

This equation is different from the Fokker-Planck equation (18) which we get from the stochastic equation (16). The difference concerns the terms $\partial_{\phi} \frac{1}{3 H}$ which must be neglected in a consistent way. An alternative procedure is to define $\tilde{P}=a^{-3} P^{r e t}$ insert it in Eq. (34) (for the integrated transition function) and take the adjoint of this equation. As a result we obtain

$$
\begin{aligned}
\partial_{t} \tilde{P}= & \frac{\gamma^{2}}{2} \partial_{X} a^{-\frac{3}{2}} \frac{1}{3 H} \partial_{X} a^{-\frac{3}{2}} \frac{1}{3 H} \tilde{P} \\
& +\frac{9}{8 \pi^{2}} \partial_{X} H^{\frac{5}{2}} \frac{1}{3 H} \partial_{X} H^{\frac{5}{2}} \frac{1}{3 H} \tilde{P} \\
& +\partial_{X}\left(V^{\prime} \frac{1}{3 H} \tilde{P}\right)
\end{aligned}
$$

which coincides with Eq. (18).

In this way we have achieved the slow-roll approximation of the stochastic system (9), (12) on the basis of probability distributions. The approximation could be controlled by estimating the neglected terms of the exact probability distributions (28), (29).

\section{Application of some general results on diffusions}

We consider a diffusion equation of the form

$d \xi=-\beta(\xi) d t+\sigma(\xi) \circ d B=-\tilde{\beta} d t+\sigma d B$ 
where $\sigma d B$ is Ito differential [21] and

$\tilde{\beta}=\beta-\frac{1}{2} \sigma^{\prime} \sigma$

In general, solutions of Eq. (37) exist till the random explosion time $\tau$. Nevertheless, probability distributions of Sect. 6 (satisfying the Kolmogorov equations) are well defined for arbitrary time as conditional probabilities $P_{t}(A) \equiv P\left(\xi_{t} \in\right.$ $A \mid t<\tau(\xi))$. There is a useful criterion [27] when the stochastic process $\xi$ can be defined for arbitrarily large time. Define a Lyapunov function $L(x)$ as a twice differentiable positive function increasing to $+\infty$ when $|x| \rightarrow \infty$ and satisfying the inequality

$\mathcal{A} L<-\alpha L+r$

where $\alpha$ and $r$ are positive constants and $\mathcal{A}$ is the differential operator on the rhs of the retrospective Kolmogorov equation. The basic theorem of diffusion processes states [27] that if for the given diffusion process (defined by $\mathcal{A}$ ) there exists a Lyapunov function then the process can be defined for arbitrarily large time.

Let us consider as a simple example $\sigma=$ const. Take $L=x^{2}$, then for the process (37)

$\mathcal{A} L=\sigma^{2}-2 \beta x$

If $2 \beta x \geq \alpha x^{2}-r$ with $r \geq 0$ then the inequality (38) is satisfied. It holds true if $\beta=V^{\prime}$ with $V=x^{2 n}$ and fails for $V=x^{2 n-1}($ natural $n)$.

Next, we can calculate the asymptotic probability distribution. If $\tilde{\beta}$ is a continuous function, $\sigma$ is continuously differentiable and $\sigma^{2}>0$ then the Fokker-Planck equation has a unique solution. If $\int f_{*}=1$ where

$f_{*}=K \sigma^{-2} \exp \left(-\int 2 \tilde{\beta} \sigma^{-2}\right)$

with a certain finite $K>0$ then for any bounded normalized $f \geq 0$ there exists the unique limit $f_{*}$ when $t \rightarrow \infty$ of the Markov semigroup $P_{t} f[27,28]$. This $f_{*}$ is called the stationary probability distribution. In our case

$\frac{1}{2} \sigma^{2}=\frac{1}{18} \frac{\gamma^{2}}{a^{3} H^{2}}+\frac{1}{8 \pi^{2}} H^{3}$

and $\beta=\frac{1}{3 H} V^{\prime}$. In most models of inflation if $\gamma^{2}>0$ (the thermal noise is non-zero) then $\sigma^{2}>0$. If there is no thermal noise then $\sigma \simeq 0$ if $H^{2} \simeq V \simeq 0$ what happens in the examples of Sect. 5. Hence, without the thermal noise there may be difficulties with the limit $t \rightarrow \infty$.

\section{Stationary probability distribution of the inflaton}

The stationary probability $P_{\infty}(\phi)$ is the limit of $P_{t}$ for $t \rightarrow \infty$ [28]. In the Stratonovitch interpretation it can be obtained from the requirement $\partial_{t} P_{\infty}=0$ which gives (after an integration over $\phi$ )

$$
\begin{aligned}
& \frac{\gamma^{2}}{18} \frac{1}{H a^{\frac{3}{2}}} \partial_{\phi} \frac{1}{H a^{\frac{3}{2}}} P_{\infty}+\frac{1}{8 \pi^{2}} H^{\frac{3}{2}} \partial_{\phi} H^{\frac{3}{2}} P_{\infty} \\
& \quad+(3 H)^{-1} V^{\prime} P_{\infty}=0 .
\end{aligned}
$$

The stationary solution of Eq. (41) without the StarobinskyVilenkin noise is (from Eq. (39))

$$
\begin{aligned}
& P_{\infty}=\sqrt{V} \exp \left(-12 \pi G \int^{\phi} d \phi^{\prime}\left(V^{\prime}\right)^{-1} V\right) \\
& \exp \left(-\frac{6}{\gamma^{2}} \sqrt{\frac{8 \pi G}{3}} \int d \phi V^{\prime} \sqrt{V}\right. \\
& \left.\exp \left(-24 \pi G \int^{\phi} d \phi^{\prime}\left(V^{\prime}\right)^{-1} V\right)\right),
\end{aligned}
$$

where the exponential factors in Eq. (42) come from the formula for $a^{3}$ (Eq. 15). For a large $|\phi|$ we have $a(\phi) \rightarrow 0$ in most of our models of Sect. 5. Hence, we can neglect the $\gamma^{-2}$ term. Then,

$P_{\infty} \simeq \sqrt{V} \exp \left(-12 \pi G \int^{\phi} d \phi^{\prime}\left(V^{\prime}\right)^{-1} V\right)$

If $\gamma=0$ (the thermal noise is absent) then we obtain the Starobinsky solution (discussed also by Vilenkin [12] and Linde [29,30])

$P_{\infty}=V^{-\frac{3}{4}} \exp \left(\frac{3}{8 G^{2}} \frac{1}{V}\right)$.

The solution (44) is not normalizable if $V \geq 0$ and $V=0$ at a certain $\phi$ or $V$ does not decay fast enough for large $\phi$. The authors $[13,14]$ impose boundary conditions excluding the regions where $P_{\infty}$ (44) is not integrable. Then, one has to study whether the dependence on boundary conditions has some consequences on calculated expectation values.

With $\gamma \neq 0$ in Eq. (41) we write

$\tilde{P}=H^{-1} a^{-\frac{3}{2}} P_{\infty}$

Then, the equation for $\tilde{P}$ is

$\frac{\gamma^{2}}{18} H^{-1} a^{-\frac{3}{2}} \partial_{\phi} \tilde{P}+\frac{1}{8 \pi^{2}} H^{\frac{3}{2}} \partial_{\phi}\left(H^{\frac{5}{2}} a^{\frac{3}{2}} \tilde{P}\right)=-\frac{1}{3} V^{\prime} a^{\frac{3}{2}} \tilde{P}$.

Using the formulas for $H$ and for $a$ (Eq. (15)) we obtain

$$
\begin{aligned}
\ln \tilde{P}= & -6 \int d \phi H a^{3}\left(\gamma^{2}+\frac{9}{4 \pi^{2}} H^{5} a^{3}\right)^{-1} \\
& \times\left(V^{\prime}+\frac{10}{3} G^{2} V V^{\prime}-32 \pi G^{3} V^{3}\left(V^{\prime}\right)^{-1}\right) .
\end{aligned}
$$

If $V^{\prime} \geq 0$ and $\left(1+\frac{10}{3} G^{2} V-32 \pi G^{3} V^{3}\left(V^{\prime}\right)^{-2}\right) \geq 0$ then $\tilde{P}$ improves integrability and we can use the saddle point method for calculations. If $\ln \tilde{P} \geq \gamma^{-2} K$ with a certain constant $K$ then for any $f$ we have 
$\left|\int f P_{\infty}\right| \leq \exp \left(\gamma^{-2} K\right) \int|f| H a^{-\frac{3}{2}}$. This estimate can be sufficient for integrability. In general, we have to find a $\phi$ dependent upper bound on $\ln \tilde{P}$ in order to prove integrability.

For most potentials of Sect. $5 a \rightarrow 0$ for $|\phi| \rightarrow \infty$ then $a^{3} H^{5} \rightarrow 0$. Hence, we get from Eqs. (45), (46) $P_{\infty} \simeq H a^{\frac{3}{2}}$ at large $\gamma$ coinciding with the formula (43). As an explicit example we consider the chaotic inflation potential $V=g \phi^{2 n}$. The integrand $\ln \tilde{P}$ in Eq. (46) is a bounded function of $\phi$ because $a \simeq \exp \left(-2 \pi G n^{-1} \phi^{2}\right)$.Then, the formula (45) gives for a large $|\phi|($ small $a)$

$P_{\infty} \simeq|\phi|^{n} \exp \left(-3 \pi G n^{-1} \phi^{2}\right)$.

At small $\phi$ we have $a^{3} H^{5} \rightarrow 0$. So, the formula (43) is applicable for small as well as large $\phi$. The Starobinsky formula (44) (obtained without the thermal noise) gives $P_{\infty}$ which is not integrable at $\phi=0$.

When $a^{3} H^{5} \rightarrow \infty$ and we neglect $\gamma^{2}$ in Eq. (46) then

$$
\begin{aligned}
\ln \tilde{P} \simeq & -\frac{8 \pi^{2}}{3} \int d \phi H^{-4} \\
& \times\left(V^{\prime}+\frac{10}{3} G^{2} V V^{\prime}-32 \pi G^{3} V^{3}\left(V^{\prime}\right)^{-1}\right) .
\end{aligned}
$$

We can calculate the rhs of Eq. (48) and convince ourselves that in this case we reach the Starobinsky formula (44). In the estimates of integrals with respect to $P_{\infty}$ in the next section we cannot let $\gamma^{2} \rightarrow 0$.

\section{Probabilistic estimates of the slow-roll regime}

We would like to estimate on the basis of the Fokker-Planck equation (18) the probability that $\left|\partial_{t}^{2} \phi\right|<<\left|3 H \partial_{t} \phi\right|$. As a consequence of the (deterministic) equations of Sect. 2 we can calculate

$\left\langle\partial_{t}^{2} \phi\left(3 H \partial_{t} \phi\right)^{-1}\right\rangle=\frac{1}{3}\langle\tilde{\epsilon}-\tilde{\eta}\rangle$.

In the classical EKG equations of Sect. 3 the regime of small $\tilde{\epsilon}$ and $\tilde{\eta}$ is closely related with the inflation regime according to Eq. (5). We can study both problems in probabilistic terms by a calculation of the mean values of the acceleration and the derivatives of the inflaton (as in Eq. (49)). So, according to Eq. (5) (strictly speaking this criterion applies if the rhs of Eq. (49) is small)

$\left\langle\partial_{t}^{2} a\right\rangle=\left\langle a H^{2}\right\rangle-\left\langle a H^{2} \tilde{\epsilon}\right\rangle$.

The inequality $\left\langle a H^{2}\right\rangle>\left\langle a H^{2} \tilde{\epsilon}\right\rangle$ depends on the probability distribution of $\phi$ determined by the stochastic equation (16). However, without the deterministic inflation the stochastic approximation to the quantum noise is hard to justify. One should rather consider Eq. (50) as a condition for the preservation of inflation by quantum and thermal fluctuations.
In order to calculate the probability distribution of the variables in Eqs. (49), (50) we would need to solve the stochastic equations or the Fokker-Planck equations. We are able to calculate only the mean values (49), (50) at large time using the stationary probability distribution. Nevertheless, such calculations give some hints on the role of thermal and quantum fluctuations in the evolution of inflation. So, in the model $V=g \phi^{2 n}(n>1)$ with the thermal noise we have on the basis of the approximation(47)

$$
\begin{gathered}
\langle\tilde{\epsilon}\rangle=4 n^{2}(16 \pi G)^{-1} \int d \phi|\phi|^{n-2} \exp \left(-\frac{3 \pi G}{n} \phi^{2}\right) \\
\times\left(\int d \phi|\phi|^{n} \exp \left(-\frac{3 \pi G}{n} \phi^{2}\right)\right)^{-1}=\frac{2 n}{n-1}
\end{gathered}
$$

and

$\langle\tilde{\eta}\rangle=\frac{2(2 n-1)}{n-1}$

So, $\langle\tilde{\epsilon}\rangle$ and $\langle\tilde{\eta}\rangle$ are never small. The reason is that in the $\phi^{2 n}$ models $\langle\tilde{\epsilon}\rangle$ and $\langle\tilde{\eta}\rangle$ behave as $n^{2} \phi^{-2}$. The probability distribution of the stochastic process $\phi_{t}$ gives big weight to small values of $\phi$. For this reason the expectation values of $\langle\tilde{\epsilon}\rangle$ and $\langle\tilde{\eta}\rangle$ cannot be small. As a consequence the approximation on which the stochastic equation (16) is based does not seem to be applicable. Nevertheless, if we still insist on applying Eq. (50) then for large $n$ (owing to the term $H^{2} \simeq \phi^{2 n}$ )

$\left\langle\partial_{t}^{2} a\right\rangle \simeq\left\langle a|\phi|^{n}\right\rangle-\left\langle a|\phi|^{n} \tilde{\epsilon}\right\rangle>0$

because the expectation value $\left\langle|\phi|^{r}\right\rangle$ is an increasing function of $r$.

Let us consider next the double-well potential (26). The Starobinsky formula (44) does not give an integrable stationary probability because $\frac{1}{V}$ is singular at $g \phi^{2}=\mu^{2}$. If the quantum noise is absent then

$$
\begin{aligned}
P_{\infty}= & H a^{\frac{3}{2}} \exp \left(-\gamma^{-2} g \sqrt{24 \pi G g}\right. \\
& \times \int d \phi\left|\phi^{2}-\frac{\mu^{2}}{g}\right|\left(\phi^{2}-\frac{\mu^{2}}{g}\right) \phi|\phi|^{\frac{6 \pi G \mu^{2}}{g}} \\
& \left.\times \exp \left(-3 \pi G \phi^{2}\right)\right)
\end{aligned}
$$

where

$H a^{\frac{3}{2}}=\sqrt{\frac{2 \pi G g}{3}}\left|\phi^{2}-\frac{\mu^{2}}{g}\right||\phi|^{\frac{3 \pi G \mu^{2}}{g}} \exp \left(-\frac{3}{2} \pi G \phi^{2}\right)$

for a large $\gamma$ the last factor in Eq. (53) is irrelevant. It can be seen thaat $\langle\tilde{\epsilon}\rangle$ and $\langle\tilde{\eta}\rangle$ are infinite because of the singularity of the integral at $\phi^{2}=\frac{\mu^{2}}{g}$. Nevertheless, we can calculate for large $\gamma$ 


$$
\begin{aligned}
& \left\langle\partial_{t}^{2} a\right\rangle=\frac{8 \pi G}{3} \int\left(\left(\phi^{2}-\frac{\mu^{2}}{g}\right)^{2}-\frac{\phi^{2}}{8 \pi G}\right) \\
& \quad \times|\phi|^{\frac{5 \pi G \mu^{2}}{g}} \exp \left(-\frac{5}{2} \pi G \phi^{2}\right) \\
& \quad \times\left(\int\left|\phi^{2}-\frac{\mu^{2}}{g}\right||\phi|^{\frac{3 \pi G \mu^{2}}{g}} \exp \left(-\frac{3}{2} \pi G \phi^{2}\right)\right)^{-1}
\end{aligned}
$$

$\left\langle\partial^{2} a\right\rangle$ is positive (because $\int x^{\alpha} \exp \left(-x^{2}\right)$ is an increasing function of $\alpha$ ). If we calculate the integral (53) at $\gamma \rightarrow 0$ by means of the saddle point method then there are saddle points at $\phi=0$ and at $\phi= \pm \frac{\mu}{\sqrt{g}}$. The saddle points give zero to the first term in Eq. (50) (and the corresponding term in the integral (53)) and non-zero contribution to the second term. Hence, acceleration can be negative for a small $\gamma \cdot\langle\tilde{\epsilon}\rangle$ and $\langle\tilde{\eta}\rangle$ are finite in a model with the double-well $(26)(n \geq 4)$. Then, we have

$$
\begin{aligned}
& \tilde{\epsilon}=\frac{n^{2}}{4 \pi G} \phi^{2}\left(\phi^{2}-\frac{\mu^{2}}{g}\right)^{-2} \\
& \tilde{\eta}=2 n \frac{(2 n-1) \phi^{2}-\frac{\mu^{2}}{g}}{4 \pi G}\left(\phi^{2}-\frac{\mu^{2}}{g}\right)^{-2}
\end{aligned}
$$

and for a large $\gamma$

$$
\begin{aligned}
P_{\infty} \simeq\left|\phi^{2}-\frac{\mu^{2}}{g}\right|^{\frac{n}{2}} a= & \left|\phi^{2}-\frac{\mu^{2}}{g}\right|^{\frac{n}{2}}|\phi|^{\frac{6 \pi G \mu^{2}}{n g}} \\
& \times \exp \left(-\frac{3 \pi G}{n} \phi^{2}\right)
\end{aligned}
$$

If $n \geq 4$ then the singularity of $\tilde{\epsilon}$ (and the same singularity of $\tilde{\eta}$ ) are cancelled by the factor in $P_{\infty}$ what makes the expectation values of $\tilde{\epsilon}$ and $\tilde{\eta}$ finite. For a large $\gamma$ we may use the approximate formula (57) for calculation of expectation values. Then

$$
\begin{aligned}
\langle\tilde{\epsilon}\rangle= & \int d y e^{-y}\left|y-\frac{3 \pi G \mu^{2}}{n g}\right|^{\frac{n}{2}-2} y^{\frac{1}{2}+\frac{3 \pi G \mu^{2}}{n g}} \\
& \times\left(\int d y e^{-y}\left|y-\frac{3 \pi G \mu^{2}}{n g}\right|^{\frac{n}{2}} y^{-\frac{1}{2}+\frac{3 \pi G \mu^{2}}{n g}}\right)^{-1}
\end{aligned}
$$

For a large $\frac{G \mu^{2}}{n g}$ we obtain

$$
\langle\tilde{\epsilon}\rangle \simeq \frac{n^{2} g}{4 \pi G \mu^{2}}
$$

and a similar result for $\langle\tilde{\eta}\rangle$. Hence, both $\langle\tilde{\epsilon}\rangle$ and $\langle\tilde{\eta}\rangle$ are small if $\gamma$ and $\frac{G \mu^{2}}{n g}$ are large.

$\langle\tilde{\epsilon}\rangle$ and $\langle\tilde{\eta}\rangle$ can also be small for small $\gamma$. In fact, for $n>4$ these expectation values can be arbitrarily small when $\gamma \rightarrow 0$ as can be seen from calculations by the saddle point method. It can also be shown that for $n \geq 4$ the mean value of the acceleration is positive for large $\gamma$ (this result could be treated as an argument for an eternal inflation [1,31])and negative for small $\gamma$.

As a next example (when the Starobinsky formula (44) does not give a stationary probability) let us consider $V=$ $g \exp (\lambda \phi)$. If only the thermal noise is present then (according to Eqs. (42) and (22))

$$
\begin{aligned}
P_{\infty}= & \exp \left(\left(\frac{\lambda}{2}-\frac{12 \pi G}{\lambda}\right) \phi\right) \\
& \times \exp \left(-2 \gamma^{-2} \sqrt{96 \pi G g}\left(3 \lambda-\frac{48 \pi G}{\lambda}\right)^{-1}\right. \\
& \left.\times \exp \left(\left(\frac{3}{2} \lambda-\frac{24 \pi G}{\lambda}\right) \phi\right)\right)
\end{aligned}
$$

$\int P_{\infty}$ is finite if $\lambda^{2}>24 \pi G$. With both quantum and thermal noises we must ensure for integrability that the last term in the brackets (46) is positive. This will be the case if

$$
\left(\frac{10}{3} \lambda G^{2}-\frac{32 \pi G^{3}}{\lambda}\right) \exp (2 \lambda \phi)>0
$$

Hence, $\lambda^{2}>9,6 \pi G$ (ensures the previous requirement $\lambda^{2}>$ $24 \pi G)$. Then, $\tilde{\epsilon}=\frac{1}{16 \pi G} \lambda^{2}>\frac{3}{2}$ and $\tilde{\eta}>3$ so that in Eq. (49) $|\tilde{\eta}-\tilde{\epsilon}|>\frac{3}{2}$. The mean acceleration (50) is negative for $\lambda^{2}>24 \pi G$ (for these values of $\lambda^{2}$ it is also negative in the exact deterministic model (9)-(12) [32]). However, because $\tilde{\epsilon}$ and $\tilde{\eta}$ with the quantum noise are not small the application of the diffusive approximation to the quantum noise and at the next step a reduction of Eq. (6) to Eq. (16) cannot be justified.

In the case of the Starobinsky potential (21) [25] according to Eq. (22)

$$
\begin{aligned}
P_{\infty}= & |\exp (\lambda \phi)-1| \exp \left(-\frac{6 \pi G}{\lambda} \phi-\frac{6 \pi g}{\lambda^{2}} \exp (-\lambda \phi)\right) \\
& \times \exp (\ln \tilde{P})
\end{aligned}
$$

We obtain that $\ln \tilde{P}$ is bounded for negative $\phi$. For positive $\phi$ we have $\ln \tilde{P}<\frac{K}{\lambda \gamma^{2}} \phi$ with $K>0$. Hence, $P_{\infty}$ is integrable for a sufficiently large $\lambda$ (depending on $\gamma$ ). $\langle\tilde{\epsilon}\rangle$ and $\langle\tilde{\eta}\rangle$ are infinite. $\left\langle\partial_{t}^{2} a\right\rangle$ is finite and negative for large $\lambda$. We get finite $\langle\tilde{\epsilon}\rangle$ and $\langle\tilde{\eta}\rangle$ if instead of the Starobinsky potential we take

$V=g(\exp (\lambda \phi)-1)^{n}$

with $n \geq 4$.

For the natural inflation (23) the Starobinsky stationary distribution (44) does not exist because of the singularity of $\frac{1}{V}$ at $\lambda \phi=\pi$. The stationary probability distribution (45), (46) is well-defined and integrable because $a^{3} H^{5}$ is bounded. $\langle\tilde{\epsilon}\rangle$ and $\langle\tilde{\eta}\rangle$ are infinite as a consequence of the singularity of $\tilde{\epsilon}$ and $\tilde{\eta}$ at $\lambda \phi=\pi$. The acceleration of Eq. (50) is (up to a positive normalization constant) 


$$
\begin{aligned}
\left\langle\partial^{2} a\right\rangle= & \frac{8 \pi g G}{3} \int_{-\frac{\pi}{\lambda}}^{\frac{\pi}{\lambda}} d \phi\left(\left|\sin \left(\frac{\lambda \phi}{2}\right)\right|^{\frac{24 \pi G}{\lambda}}\right. \\
& \left.-\left(1+\frac{\lambda^{2}}{96 \pi G}\right)\left|\sin \left(\frac{\lambda \phi}{2}\right)\right|^{\frac{24 \pi G}{\lambda}+2}\right) \exp (\ln \tilde{P})
\end{aligned}
$$

where we applied the notation of Eq. (46). $\ln \tilde{P}$ is a regular bounded function hence its contribution to the integral is negligible (at large $\gamma$ ). The $P_{\infty}$ integral of powers of $\sin ^{2 \mu-1}$ is equal to $2^{2 \mu-2} \Gamma(\mu)^{2}(\Gamma(2 \mu))^{-1}$. Hence, $\left\langle\partial^{2} a\right\rangle$ is negative for large $\lambda$. It can be seen from our calculations that if instead of the potential (23) we considered

$V=g(1+\cos (\lambda \phi))^{n}$

with $n \geq 2$ then expectation values of $\tilde{\epsilon}$ and $\tilde{\eta}$ would be finite as follows from the approximation (43) for the stationary probability distribution

$P_{\infty} \simeq\left|\cos \left(\frac{\lambda \phi}{2}\right)\right|^{n}\left|\sin \left(\frac{\lambda \phi}{2}\right)\right|^{\frac{24 \pi G}{\lambda^{2}}}$

Then the singularity of $\tilde{\epsilon}$ and $\tilde{\eta}$ cancels with the corresponding factor in $P_{\infty}$.

\section{Summary and conclusions}

The slow-roll approximation is a useful tool in the study of deterministic as well as stochastic EKG systems. In the deterministic case we stop using it as soon as the parameters (functions of $\phi) \tilde{\epsilon}$ and $\tilde{\eta}$ become large. In the stochastic version we should argue that we stop applying slow-roll for a time $t$ such that the probability that e.g. $\tilde{\epsilon}>\frac{1}{2}$ and $\tilde{\eta}>\frac{1}{2}$ becomes large. This time is difficult to estimate. As in the theory of Brownian motion [3] we suggest that with the strong "friction" $H$ the first order equation (16) may be a reliable approximation to the second order differential equation (6). However, for a stochastic approximation of the quantum noise the requirement of small $\tilde{\epsilon}$ and $\tilde{\eta}$ seems unavoidable. We distinguish a class of models where the expectation values of these inflation parameters are indeed small. We calculate expectation values of $\tilde{\epsilon}, \tilde{\eta}$ and $\partial_{t}^{2} a$ with respect to the stationary probability which gives the probability distribution for a large time. We show that in some models the acceleration can be positive showing that quantum and thermal fluctuations do not destroy inflation even at large time. The sign of the acceleration can depend on the friction as we have shown in the double-well model.

Data Availability Statement This manuscript has no associated data or the data will not be deposited. [Author's comment: This manuscript has no associated data.]
Open Access This article is distributed under the terms of the Creative Commons Attribution 4.0 International License (http://creativecomm ons.org/licenses/by/4.0/), which permits unrestricted use, distribution, and reproduction in any medium, provided you give appropriate credit to the original author(s) and the source, provide a link to the Creative Commons license, and indicate if changes were made.

Funded by $\mathrm{SCOAP}^{3}$.

\section{Appendix:e-fold time}

In the calculation of the power spectrum [33,34] in order to take into account fluctuations of the quantum gravitational field the use of the e-fold time $v$ defined by

$v=\int H d t$

is crucial. The change of time does not have substantial role in the discussion in this paper (because we discuss solely the fluctuations of the $\phi$-field) as can be seen from the basic equations expressed in the e-fold time. Eq. (16) takes the form

$d \phi=-\frac{V^{\prime}}{3 H^{2}} d \nu+\frac{\gamma}{3 H^{\frac{3}{2}}} a^{-\frac{3}{2}} \circ d B+\frac{1}{2 \pi} H \circ d W$,

Eq. (18)

$$
\begin{aligned}
\partial_{\nu} P= & \frac{\gamma^{2}}{18} \partial_{\phi} \frac{1}{(H a)^{\frac{3}{2}}} \partial_{\phi} \frac{1}{(H a)^{\frac{3}{2}}} P+\frac{1}{8 \pi^{2}} \partial_{\phi} H \partial_{\phi} H P \\
& +\partial_{\phi}(3 H)^{-2} V^{\prime} P .
\end{aligned}
$$

Equation (45) is

$P_{\infty}=(H a)^{\frac{3}{2}} \tilde{P}$

where $\tilde{P}$ is defined by the same formula (46). So, the change of time has a minor effect on the expectation values of $\tilde{\epsilon}, \tilde{\eta}$ and $\partial^{2} a$ but does not change substantially our conclusions.

\section{References}

1. A. Linde, Phys. Lett. B 129, 177 (1983)

2. A.A. Starobinsky, Lecture Notes in Physics, in Current Topics in Field Theory, Quantum Gravity and Strings, vol. 246, ed. by H.J. Vega, N. Sanchez (Springer, New York, 1986)

3. E. Nelson, Dynamical Theories of Brownian Motion (Princeton Univ Press, Princeton, 1967)

4. A. Berera, Li-Zhi Fang, Phys. Rev. Lett. 74, 1912 (1995)

5. A. Berera, I.G. Moss, R.O. Ramos, Rep. Progr. Phys. 72, 026901 (2009)

6. M.H. Hall, I.G. Moss, A. Berera, Phys. Rev. D 69, 083525 (2004)

7. R.O. Ramos, L.A. da Silva, JCAP03 032 (2013)

8. Z. Haba, Eur. Phys. J. C 78, 596 (2018)

9. A.D. Linde, Phys. Lett. B 116, 335 (1982)

10. A.A. Starobinsky, Phys. Lett. B 117, 175 (1982)

11. A. Vilenkin, L.H. Ford, Phys. Rev. D 26, 1231 (1982)

12. A. Vilenkin, Phys. Rev. D 27, 2848 (1983) 
13. V. Vennin, H. Assadullahi, H. Firouzjahi, M. Noorbala, D. Wands, Phys. Rev. Lett. 118, 031301 (2017)

14. H. Assadullahi, H. Firouzjahi, M. Noorbala, V. Vennin, D. Wands, JCAP06, 043 (2016)

15. Z. Haba, Int. J. Mod. Phys. D 28, 1950085 (2019)

16. V. Mukhanov, Physical Foundations of Cosmology (Cambridge, 2005)

17. A. Berera, Phys. Rev. D 54, 2519 (1996)

18. Z. Haba, Adv. High. Energy. Phys. 2018, 7204952 (2018). arXiv: 1807.00639

19. Z. Haba, in preparation

20. A.A. Starobinsky, J. Yokoyama, Phys. Rev. D 50, 6357 (1994)

21. N. Ikeda, S. Watanabe, Stochastic Differential Equations and Diffusion Processes (North Holland, 1981)

22. I.I. Gikhman, A.B. Skorohod, Stochastic Differential Equations (Springer, New York, 1972)

23. J. Martin, C. Ringeval, V. Vennin, Encyclopedia Inflationaris, arXiv: 1303.3787
24. S.V. Ketov, A.A. Starobinsky, JCAP08, 022 (2012)

25. A. Kehagias, A.M. Dizgah, A. Riotto, Phys. Rev. D 89, 043527 (2014)

26. K. Freese, J.A. Frieman, A.V. Olinto, Phys. Rev. Lett. 65, 3233 (1990)

27. R.Z. Khasminski, Stochastic Stability of Differential Equations (Sijthoff and Noordhoof, Amsterdam, 1980)

28. A. Lasota, M.C. Mackey, Probabilistic Properties of Deterministic Systems, (Cambridge, 1985)

29. A.D. Linde, Phys. Rev. D 58, 083514 (1998)

30. A.D. Linde, Phys. Rev. D 49, 748 (1994)

31. A. Vilenkin, Nucl. Phys. B. Proc. Suppl. 88, 67 (2000)

32. F. Lucchin, S. Mataresse, Phys. Rev. D 32, 1316 (1985)

33. F. Finnelli, G. Marozzi, A.A. Starobinsky, G. Venturi, Phys. Rev. D 79, 044007 (2009)

34. V. Vennin, A.A. Starobinsky, Eur. Phys. J. C 75, 413 (2015) 